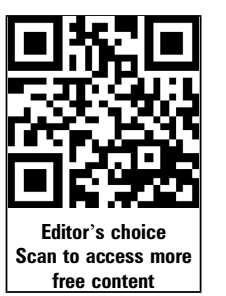

${ }^{1}$ Pharmacy and Medicines Management Centre, Northern Health and Social Care Trust, Beech House, Antrim Area Hospital, Antrim, Northern Ireland

${ }^{2}$ Clinical Pharmacy Practice Research Group, School of Pharmacy, The Queens University of Belfast, Belfast, Northern Ireland

\section{Correspondence to}

Professor Michael G Scott, Pharmacy and Medicines Management Centre, Northern Health and Social Care Trust, Beech House, Antrim Area Hospital, Antrim BT41 2RL, Northern Ireland; drmichael. scott@northerntrust.hscni.net

Received 16 October 2014 Revised 13 March 2015 Accepted 16 March 2015 Published Online First 13 April 2015

\title{
Integrated medicines management to medicines optimisation in Northern Ireland (2000-2014): a review
}

\author{
Michael G Scott, ${ }^{1}$ Claire Scullin, ${ }^{2}$ Anita Hogg, ${ }^{1}$ Glenda F Fleming, ${ }^{1}$ James C McElnay ${ }^{2}$
}

\begin{abstract}
Objective The objective of this review was to assess the improvement in medicines processes in Northern Ireland focusing mainly on secondary care and at the interface with primary care and also to consider the benefits of enabling technologies for the system.

Methods The review was undertaken by examining all of the publications that had been produced together with relevant context articles that framed and supported the requisite process changes together with a synopsis of relevant enabling technologies that were devised. Key outputs were summarised and incorporated into the system review.
\end{abstract}

Results The optimisation of the medicines process has led to benefits to patients in terms of morbidity and mortality in addition to a reduction in healthcare resource utilisation. Key findings include reduced length of stay, reduced readmission rates, improved Medicines Appropriateness Index, improved Medicines Administration Error rate, positive impact on Risk Adjusted Mortality Index and improved communication across the primary/secondary care interface. Enabling technology solutions were also implemented.

Conclusions The journey from medicines management to optimisation has resulted in significant improvements in the quality and safety of medicines yielding health gain and economy and a return on investment of $£ 5-8$ for each $\mathrm{f} 1$ invested. The complementary role of pharmacy in the multidisciplinary team has been identified and evidenced. The next steps will be to further integrate with primary care and deliver improvements in medicines processes in that sector together with requisite enabling technologies.

\section{INTRODUCTION}

Pharmacy is the healthcare profession that is responsible for ensuring safe, effective and rational use of medicines; thus, it plays a vital role in healthcare delivery worldwide. ${ }^{1}$ Current healthcare systems continue to face great challenges due to increasing numbers of adverse events, poor adherence, increasing numbers of medication incidents and inadequate communication at transitions of care, especially at the primary/secondarycare interface.

Expenditure on medicines is the second largest cost in healthcare with an excess of $£ 550$ million CrossMark

To cite: $\mathrm{Scott} M G$,

Scullin C, Hogg A, et al. Eur $J$ Hosp Pharm

2015;22:222-228. being spent per annum by the Health and Personal Social Services in Northern Ireland, accounting for almost $13 \%$ of the whole healthcare budget.

In 2000 the National Health Service put out the challenge to pharmacy in the document 'Pharmacy in the Future-Implementing the National Health Service (NHS) Plan', to meet the changing needs of patients. $^{2}$ To do this pharmacy needed to make sure that patients can get medicines or pharmaceutical advice easily, make sure that patients get more support in using their medicines, and give patients the confidence that they are getting good advice when they consult a pharmacist.

Research then emanated from the National Audit Commission Report 'A Spoonful of Sugar' 3 and 'An Organisation with a Memory' ${ }^{4}$ in attempting to integrate these recommendations across the continuum action that is Medicines Management. Medicines Management has been defined by the Audit Commission as:

"Encompassing the entire way that medicines are received, procured, delivered, prescribed, administered and reviewed, to optimise the contribution that medicines make to producing informed and desired outcomes of patient care". It encompasses all aspects of medicines use from prescribing of medicines through the ways in which medicines are taken or not taken by patients. ${ }^{5}$ Medicines management involves the systematic provision of medicine therapies through a partnership of effort between patients and professionals to deliver best patient outcomes and minimise cost. ${ }^{6}$ The quality use of medicines is a key factor in achieving positive health outcomes.

Thus there were a number of key deficiencies in the system: inefficient procurement, lack of compliance with prescribed therapies, morbidity and mortality, wastage of medicines, medication errors, adverse drug reactions, intrasector and intersector transitions of care, multidisciplinary teamwork and pharmacy skill-mix.

It was against this background that the integrated medicines management (IMM) programme was commenced in Northern Ireland in 2000. Over the time frame of this work there were other recognised system changes that would yield patient benefit, with one such being One Stop Dispensing which refers to the dispensing of inpatient and discharge medicines in a single supply on admission already labelled with instructions for the patient. It is currently referred to as 'dispensing for discharge'. Patients are encouraged to bring their own medicines into hospital on admission and medicines assessed by the pharmacy staff as suitable for use are used by the patient during their stay. A 28 day supply is given of any of the patients medicine deemed unsuitable for use, when the quantity of a particular medicine is depleted and when new medicines are commenced, all medicines being kept in the patient's locker. This was a requirement of the National Service Framework for Older People. ${ }^{8}$ 
Further, over the time period being reported, there was involvement with the Institute of Healthcare Improvement under The Safer Patient Initiative. In relation to the medicines management component of the work, the terminology of medicines reconciliation was introduced. ${ }^{9}$ Medicines reconciliation is a process of identifying the most accurate list of all medications a patient is taking, including name, dosage, frequency and route and then using this list to provide the correct medications for patients in hospital. Reconciliation involves comparing this list against the doctors prescribing on admission, transfer or discharge, with reasons for any omissions or dose changes being documented. This concept is carried right through all stages of the patient journey in hospital in this Integrated Medicines Management (IMM) work.

This has been an ongoing process since that time, but now with a change in terminology to medicines optimisation with a the Royal Pharmaceutical Society in their recent report 'Good Practice Guidance Document' stated that 'Medicines optimisation is about ensuring that the right patients get the right choice of medicines at the right time". By focusing on patients and experiences the goal is to help patients to improve their outcomes, take their medicines correctly, avoid taking unnecessary medicines and improve medicines safety. Ultimately medicines optimisation can help encourage patients to take ownership of their treatment. ${ }^{10}$

The predicted outcomes of the service were:

1. Improved patient safety through the appropriate and optimal use of their medicines

2. Improved bed utilisation through reduced cost per episode and reduced readmission rate

3. Improved utilisation of medical, nursing and pharmacy staff, thereby alleviating the workload pressures

4. Reduced medicines costs through decreased wastage and improved procurement mechanisms.

\section{IMPLEMENTATION}

This initial work entailed an enhanced clinical skills training programme for pharmacists and technicians. The new medicines management process then entailed comprehensive pharmacy teams involved at admission, inpatient stay and at discharge, incorporating communication at the intrasector transitions at admission and discharge where most medicine-related problems occur.

\section{Admission}

At admission an acute drug history was obtained, based upon histories taken from the general practitioner (GP) record, admission prescription list (Kardex), patient's own drugs, as well as the patient/case drug history. Further, where the patient reported using the same community pharmacy on a regular basis (ie, at least $75 \%$ of the time) to have her medicines dispensed, then the community pharmacy used was also included.

Pharmacy technicians also used an algorithm at the time of admission to assess the supply and suitability of patients' own drugs for return to the patient if required at discharge. Technicians also had an intervention form and a referral system for queries to the relevant clinical pharmacist.

At admission the following tasks were undertaken:

- Construct an acute drug history

- Deal with discrepancy

- Carry out a medication review to compile an acute Kardex

- Assess patients' own drugs

- Communicate with primary care regarding the drug history (phone/fax), allergy/side-effects

- Obtain other relevant patient information

- Clarify queries

\section{Inpatient}

Inpatient monitoring tasks included:

- Full medication review

- Monitor drug therapy

- Monitor efficiency

- Monitor for continuing indications

- Monitor for tolerance

- Monitor for adverse events

- Monitor relevant laboratory tests

- Educate the patient on all medications

- Provide written information, for example, anticoagulant leaflet.

\section{Discharge}

The pharmacist generated and authorised the discharge following the authorisation of the immediate clinical discharge summary by the physician which was carried out under a protocol agreed by the Northern Area Prescribing Forum. After discussion with the patient the technician assessed which drugs required dispensing, taking into account any patient's own drugs which had been stored on admission.

A medicines record sheet was completed and the discharge summary was faxed to the patient's GP and nominated community pharmacist.

The key actions were:

- Prepare and sign off the discharge

- Detail medication changes or other information relevant to the GP

- Dispense only the medication required

- Provide discharge education, including a medicines record sheet

- Fax discharge summary to the GP at the community pharmacist on the day of discharge

The delivery of this service was targeted at patients based on work previously carried out within the Trust to develop a risk model for adverse drug events in the elderly. ${ }^{11}{ }^{12}$ They are described in box 1 .

\section{Medication appropriateness}

The appropriate use of medicines is paramount for effective care and therefore, to enable this to be assessed, the Medicines Appropriateness Index (MAI) tool developed by Hanlon et $a l^{13}$ was used in this work.

\section{Medicines administration}

A Medicine Administration Error (MAE) is a dose administered or omitted that deviates from that prescribed and can be due to system and/or human failure. ${ }^{14}$ Review of the literature demonstrates that the effectiveness and quality of a medicines administration system can be successfully measured by determining the

Box 1 Patient inclusion criteria for integrated medicines management

Prescribed four or more regular medications

- Prescribed a high risk or Amber List drug, for example, warfarin, digoxin, amiodarone, etc.

- Prescribed intravenous antibiotics on admission to hospital

- Taking antidepressant medications and aged 65 years or over

Previous hospital admission within the last 6 months 
incidence of MAEs. ${ }^{15}$ Hence part of the work undertaken was to measure this factor following the process change.

\section{Antimicrobial stewardship}

Optimising the use of antimicrobial agents is a critical task and given this significance an assessment of the impact of the process changes was also undertaken for these agents.

\section{Interventions}

The recording of clinical pharmacist interventions has always been important from the viewpoint of identifying errors as well as providing objective evidence of the value of clinical pharmacy services. In this work the system described by Eadon ${ }^{16}$ was used (table 1).

\section{Procurement}

Historically the approach taken to reduce medicines expenditure has been to focus almost exclusively on costs and cost-cutting initiatives. This methodology has had only limited success as it fails to address the more fundamental aspects of the safety and quality of medicine use. Hence part of this work was to develop a new approach based on the premise that quality and safety drive health gain and economy.

\section{RESULTS}

In terms of the outcomes of this initial work the following were found: ${ }^{17}$

- Drug history at admission-reduction of 4.2 errors per patient

- Kardex monitoring-5.5 interventions per patient

- Reduced length of stay by 2 days

- Increased time to readmission-20 days

- Faster medication rounds $>25$ min per round

- Faster discharge->90 min quicker

- More accurate discharge < $1 \%$ errors compared with $25 \%$ by medical staff

- Decreased rate of readmission

A numbers needed to treat analysis showed that for every 12 patients receiving the IMM service, one readmission to hospital within 12 months of discharge would be prevented.

Analysis of the benefits of the hospital-based community liaison service in the hospital revealed that $33 \%$ of patients had medication related problems post discharge with the service being felt to be very useful by GPs and community pharmacists. ${ }^{18}$ Further evaluation work demonstrated that when this work was undertaken, the intervention group had a significant reduction in drug mismatch between drugs prescribed at discharge and taken at home, when compared with control patients. They also had a greater knowledge of their drug regime 10-14 days post discharge, and patients valued the service. ${ }^{19}$

\section{Table 1 Eadon grading system (Eadon ${ }^{16}$ )}

\begin{tabular}{ll}
\hline Intervention grade & Definition \\
\hline 1 & Detrimental to patient care \\
2 & Of no significance to patient care \\
3 & Significant but does not improve patient care \\
4 & Significant and improves the standard of patient care \\
5 & Very significant; prevents major organ failure or similar \\
6 & Potentially life-saving \\
\hline
\end{tabular}

\section{Medicines Appropriateness Index}

It was found that there was a significant improvement in the MAI score between the control and intervention groups and also between admission and discharge for the intervention group. ${ }^{20}$

\section{Interventions}

In relation to this project, a total of 1628 interventions were made in 294 patients, a mean of 5.54 per patient. The majority of interventions made were Grade 4 and above which meant that the interventions were significant, and resulted in an improvement in care.

\section{Procurement}

The use of STEPSelect (Safe Therapeutic Economic Pharmaceutical Selection) contributed significantly to the findings in the McKinsey report ${ }^{21}$ which demonstrated that the hospital prescribing in $£$ per need weighted patient was $£ 50$ (with a $16 \%$ social deprivation adjustment) compared with $£ 64$ in England (table 2).

\section{Medicines administration error}

It was found that the MAE rate and time spent on administration rounds decreased after the introduction of lockers. The MAE rate dropped from $8.3 \%$ to $1.3 \%$ and the time spent on medicines administration per patient decreased from 6.80 $\pm 5.44 \mathrm{~min}$ pre intervention to $3.03 \pm 1.87 \mathrm{~min}$ post intervention. In addition other benefits included releasing nursing time to care and enhanced opportunities for patient counselling as well as easier communication with medical staff. ${ }^{22}$ Thus this process modification led to safer and faster medicine rounds leading to significant release of nursing time to care. ${ }^{23}$

\section{Antimicrobial stewardship}

It was demonstrated that the introduction of a consultant microbiologist-led ward round with inputs from a specialist antimicrobial pharmacist improved compliance with the hospital's antibiotic policy, compliance with intravenous to oral switch (55-69\%) and a reduction in duration of intravenous antibiotic courses (6.5-6.125 days). ${ }^{24}$ In addition, when an analysis of the antimicrobial treatment of lower respiratory and urinary tract infections was undertaken, it was seen that when patients were not switched in line with guidance they remained significantly longer in hospital. This result shows a significant waste of healthcare resources in terms of bed days. ${ }^{25}$

\section{Risk modelling and mortality impact}

It was found that the number of previous emergency admissions and admission medicines, together with age-adjusted comorbidity and diuretic receipt, formed a 12-month postdischarge and/ or admission risk algorithm. It was also found that the increased number of clinical pharmacy staff, pharmacists and technicians at ward level was correlated with a reduction in the risk-adjusted mortality index. ${ }^{26}$

Table 2 f Per need weight population (McKinsey Report ${ }^{21}$ ) 


\section{ENABLING TECHNOLOGY DEVELOPMENTS}

The most critical task in medicines optimisation is the redesign of the system first and foremost, but once this has been effectively achieved, technology can then be used to further enhance the process.

\section{Locker development}

In line with the best practice recommendations in the 'A Spoonful of Sugar' report an integrated locker within the patient's bedside cabinet was designed. This was a new item and designed in conjunction with nursing, infection control and clinical engineering and was used throughout the region and outside the province. The locker also had computer-monitored access thereby enabling the maintenance of a record of opening, etc. ${ }^{27}$

\section{Safe therapeutic economic pharmaceutical selection}

This model for clinician-driven procurement was developed in conjunction with a private company to ensure that medicines selection was fundamentally based on clinically related criteria such as efficacy, safety, documented effects on clinically relevant end points, ease of administration, etc. ${ }^{28}$ This was based on a decision analysis matrix model for medicines namely the System of Objectified Judgement Analysis and Informatrix. ${ }^{29}$ This system is now fully web based and also uses e-sessions to maximise clinician engagement. In summary, it allows medicine selection within a class across a wide range of indications with a focus on clinical effectiveness and safety over cost.

\section{Electronic pharmacist intervention clinical system}

This is a software tool developed in conjunction with a local company, initially to record pharmacist interventions, as well as to collect the various data sets necessary to validate the IMM process changes. ${ }^{30}$ Previously a bar code based system had been used to facilitate the recording and analysis of intervention data. $^{31}$

The intervention scheme used was based on the scale developed by Eadon.

The data demonstrated that in over $75 \%$ of instances the intervention grade was 4 or over, showing a significant benefit from pharmacist intervention in terms of patient safety.

The system has now been developed to enable pharmacists to record their clinical activities, thereby allowing optimisation of their support into patient care. Another aspect of the system is that the automatic transfer of interventions of Grade 5 and Grade 6 into Datix is being finalised, which will greatly assist in incident reporting and learning systems with regard to medicines.

In addition, the system is used on a 1 week per month basis as a performance indicator, collecting interventions and medicines reconciliation numbers in a robust and uniform manner. This, together with other key performance indicators, is very important in terms of ensuring that the medicines management processes are running as optimally as possible.

A pharmacy technician version is also under preparation.

The tool can now also be used to monitor patient flow in terms of stage of process, that is, admission, inpatient and discharge, which is also quantified clearly, enabling resources to be quickly and efficiently moved to maintain good patient flow with regards to the medicines aspect of the process.

The system runs on tablet devices and therefore is readily available to the clinical pharmacists, along with all the other key data available, such as laboratory tests that are visible on the electronic care record (ECR).

\section{Writemed}

Medicines reconciliation at all stages of the patient journey is very important but most critically at intersector transitions of care, namely admission and discharge. In order to address the deficiencies initially a paper-based system had been developed.

However, there are a number of disadvantages with paperbased medicines reconciliation processes, including:

- Details recorded in different formats by different users

- Transmitting errors

- Problems with storage of paper records

- Access to paper records

Thus Writemed was developed in conjunction with a local company and now has over 35000 MedRec records. It currently interfaces with the Patient Administration System and $\mathrm{DM}+\mathrm{D}$ (NHS drug dictionary of drugs and medical devices). It also interfaces with Electronic Pharmacist Intervention Clinical System as alluded to above. Further, the system has a query database. The system has a number of deliverables (see box 2).

Most recently, in Northern Ireland, the ECR has been introduced enabling 'windows' to be opened to see data on the GP system, plus all of the hospital systems. The next step is to get a window opened on the ECR for Writemed.

This is the optimal way to achieve fully reconciled medicines at all points, that is, by a pharmacist-operated and pharmacistdriven system.

\section{Live automated microbiology pharmacy surveillance system}

As alluded to above, the importance of using antimicrobials safely and effectively and linking this to resistance patterns in order to minimise the development of resistant organisms, is a prime function of antimicrobial pharmacists. In order to assist in this work a novel surveillance system was devised, namely Live Automated Microbiology Pharmacy Surveillance System.

The key benefits of this system are to give access to the full archived history of microbiology laboratory data which can be analysed in real time and retrospectively to detail changing trends in microorganisms and sensitivities. Data can be analysed at all patient and ecological levels. Antimicrobial use can be assessed in real time enabling rapid changes to be made as necessary. The system also assesses compliance with the Antimicrobial Policy by consultant and appropriateness of restricted antimicrobial use.

Thus this system enables much greater optimisation of antimicrobial use by the pharmacist and microbiologist.

\section{Transferability}

Following the success of the programme and the standardisation and streamlining of the processes, the system was rolled out in line with quality improvement methodology across the province,

\section{Box 2 Advantages of Writemed Medicines}

\section{Reconciliation Software}

Real-time updates with patient details

- Medication details are uniform, clear

- Med Rec record is retained indefinitely and can be reprinted and accessed by all users

- Intervention reporting is automatic

- Primary care gets a complete list on discharge

- Discharge medication list is prepopulated from the previous admission 
and with further detailed evidence being collected within another Trust area. In terms of the impact of the roll-out it was found that the initial benefits were replicated. The key findings were reduced length of stay, reduced length of stay on readmission, tending to have a longer time to, and a reduced number of, readmissions, and in economic terms it was shown that for every 100 patients receiving IMM, the opportunity cost is $£ 135 \mathrm{~K}^{32}$

The key components of the system are shown diagrammatically in figure 1 .

\section{DISCUSSION}

The system changes over the time frame of this work have demonstrated significant improvements in a number of key parameters, in relation to patient care.

This and variations of such a system are in place in varying degrees in other parts of the UK, which is not surprising given the great similarity between the healthcare systems. However the process has also been replicated in other countries such as Sweden, in Uppsala and Lund. ${ }^{33}{ }^{34}$ It has also been demonstrated to work in the South of Ireland and Norway. ${ }^{35} 36$

The improvement in medicines management at the transition, particularly post discharge, is in line with other work, for example, Cochrane et $a l^{37}$ who found that in 45 out of 50 elderly patients there was a lack of contingency in one or more aspects between drugs taken after leaving hospital and those provided on discharge.

There were also demonstrated reductions in discharge process time as well as other benefits including releasing nursing time for patient counselling, which is in line with data previously reported. 3839

The goal of any medicines administration system is to ensure that once validated, each medicine dose is administered in accordance with the prescriber's instructions. In relation to nursing, medicine administration occupies $30-50 \%$ of nurses' time in hospital and is one of the highest risk activities that a nurse performs. ${ }^{40}$ The estimated incidences of MAEs were between $6.6 \%$ and $40.6 \%$ for all doses administered by nurses. ${ }^{41-44}$ Decentralising the medicines administration system so that medicines are kept close to the patient may enhance

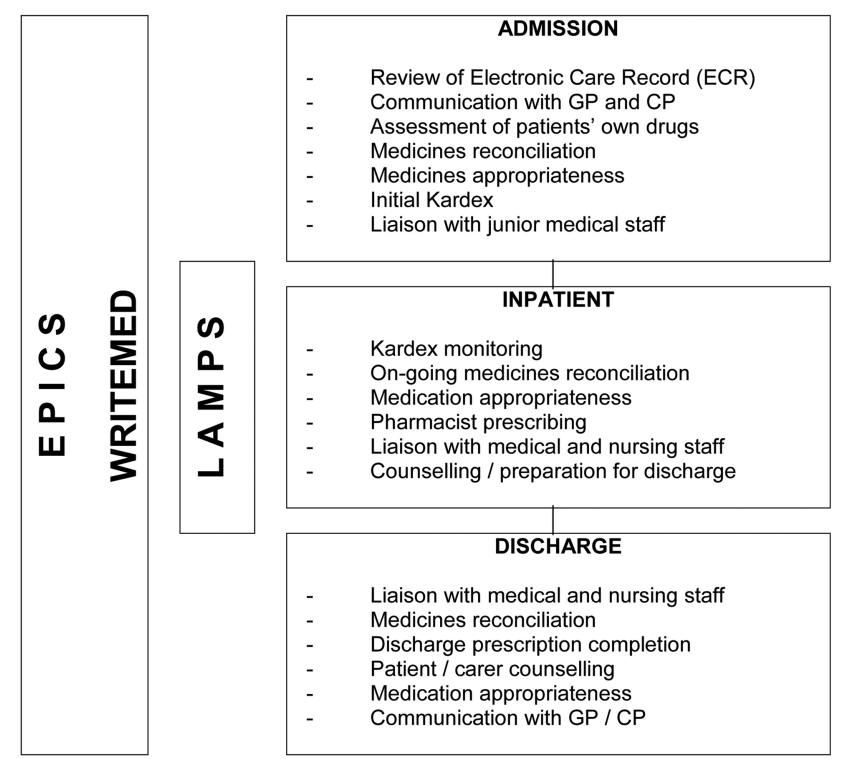

Figure 1 Key process components and technology use. safety and efficiency and minimise the number of interruptions and distractions that occur during medicines administration to a particular patient. ${ }^{45}$ The changes in the process implemented in this work in terms of further safer administration rounds are greatly reduced. MAE would validate these assertions.

In relation specifically to antimicrobial use, the results of the data with regards to improved switching from intravenous to oral, reduced costs and reduced length of stay, are in line with work published by Ramirez ${ }^{46}$ and are impartial in the context of increasing antimicrobial resistance and optimising the use of these agents. $^{47}$

There is now a lot of research ongoing into risk stratification of patients with different disease states. In relation to medicines therefore, building on earlier work, an enhanced algorithm related to post discharge and/or admission has been developed. This will be important going forward in terms of patient identification for pharmacy intervention akin to the use of the Patients At Risk of Readmission formula. ${ }^{48}$ A further key finding was the contribution to a reduction in risk-adjusted mortality index and clinical pharmacy staffing at ward level. This is in line with data from the USA regarding clinical pharmacist numbers and hospital mortality rates, which were also associated with decreased length of stay, medication errors and adverse drug reactions. ${ }^{49-51}$

The clinician driven procurement process is now well embedded and robustly supports procurement and the regional formulary, as well as being applied to wound dressings and medical and surgical devices. Its web-based operation and e-sessions methodology is unique.

\section{CURRENT FURTHER SYSTEM DEVELOPMENT DHSSPS innovation medicines management programme} Intermediate care

In relation to patients in an intermediate care setting, the role of a Consultant Pharmacist for this patient group has been evaluated.

The pilot scheme demonstrated the following benefits, namely improved MAI: a total of 1122 interventions were made in 453 patients, with $84 \%$ being Grade 4 or above, and with $42.9 \%$ of patients phoned post discharge requiring one or more interventions. $^{52}$

In addition, in health economics terms, based on the model devised by Karnon et al, ${ }^{53}$ there were significant financial benefits ranging from $£ 165000$ to $£ 280000$ per annum, yielding a return of investment (ROI) of 2.35-4.

\section{Nursing homes}

Similarly, a Consultant Pharmacist was put in place in the nursing home setting, which yielded the following key outcomes:

Improved MAI, 2.7 interventions made per patient, reduced attendances at Emergency Department and health economic gains of between $£ 165000$ and $£ 240000$ per annum, which is a ROI of $2.39-3$ per $£ 1$ million (unpublished data-in preparation).

\section{Adherence}

Currently a project is being undertaken in respect of a medicines adherence support service, linking community and hospital pharmacists and using a referral mechanism, the purpose being to identify the optimal level and method of adherence support for individual patients. 


\section{Miscellaneous}

Other system changes being evaluated include links to an independent pharmacist prescribing at all stages of the patient journey, enhanced technician roles, including a referral system, self-administration schemes, postdischarge follow-up and handover to a community pharmacist, and domiciliary care worker training with regards to medicines use.

\section{Conclusion}

The process of medicines optimisation is clearly complex and multi-factorial and therefore must incorporate a number of components in order to be effective. The process changes described here have clearly greatly improved the safety and quality of patient care resulting in health gain and economy of use of healthcare resources. While technology can assist in such change, processes must be optimised first and foremost.

The system described is transferrable and has been awarded three-star reference site status in Europe under the European Innovation Partnership on Acute and Health Ageing. ${ }^{54}$ However further system changes are still needed, particularly with regards to the suboptimal medicines management processes in primary care in order to make further progress with regards to the whole system.

\section{Key messages}

This review highlights the fact that:

- Medicines optimisation requires multi-functional interventions to be effective.

- Enabling technologies to support process re-design allows maximal use of pharmacy team resources.

- Medicines optimisation needs to be addressed in a wholesystem manner.

\section{Competing interests None.}

Provenance and peer review Commissioned; externally peer reviewed.

\section{REFERENCES}

1 Anderson $\mathrm{S}$. The state of the world's pharmacy: a portrait of the pharmacy profession. J Interprof Care 2002;16:391-404.

2 Department of Health. Pharmacy in the Future-Implementing the NHS plan. Department of Health, London 2000.

3 The Audit Commission. A spoonful of sugar-medicines management in NHS sospitals. London: The Commission, 2001.

4 Department of Health. An organisation with a memory; report of an expert group on learning from adverse events in the NHS, London 2000.

5 Simpson $D$. What medicines management means. What is medicines management and what is pharmaceutical care. Pharm J 2001;266:150.

6 Tweedie A, Jones I. What is medicines management? Pharm J 2001;266:248.

7 Dooley MJ, Allen KM, Doecke CJ, et al. A prospective mm sharing of pharmacist involved changes to drug therapy and patient management in acute care government funded hospitals. Br J Clin Pharmacol 2004;57:513-21.

8 Department of Health. National Service Framework for Older People. 2001.

9 The Institute of Healthcare Improvement (IHI). How-to Guide: Prevent Adverse Drug Events by Implementing Medication Reconciliation. 2011. http://www.ihi.org (accessed 18 Jan 2013).

10 Royal Pharmaceutical Society. Medicines Optimisation: Helping patients to make the most of medicines. May 2013.

11 McElnay JC, McCallion C, Al-Deagi F, et al. Development of a risk model for adverse drug events in the elderly. Clin Drug Investig 1997;13:47-55.

12 Morrissey EFR, McElnay JC, Scott M, et al. The influence of drugs, demographics and medical history on hospital re-admission of elderly patients. Clin Drug Investig 2003:23:119-28.

13 Hanlon JT, Schmader KE, Samsa GP, et al. A method for assessing drug therapy appropriateness. J Clin Epidermiol 1992;45:1045-51.
14 Allan E, Barker K. Fundamentals of medication error research. Am J Hosp Pharm 1990;47:555-71.

15 Taxis K, Dean B, Barber N. Hospital drug distribution systems in the UK and Germany-a study of medication errors. Pharm World Sci 1999;21:25-31.

16 Eadon $\mathrm{H}$. Ensuring the quality ward pharmaceutical interventions. Int J Pharm Pract 1992;1:145-7.

17 Scullin C, Scott MG, Hogg A, et al. An innovative approach to integrated medicines management. J Eval Clin Pract 2007;13:781-8.

18 Brookes K, Scott MG, McConnell JB. The benefits of a hospital based community service liaison pharmacist. Pharm World Sci 2000;22:33-8.

19 Bolas H, Brooke K, Scott M, et al. Evaluation of a hospital based community liaison service in Northern Ireland. Pharm World Sci 2004;26:114-20.

20 Burnett KM, Scott MG, Fleming GF, et al. Effectiveness of an integrated medicines management programme on medication appropriateness in hospitalised patients. Am J Health Syst Pharm 2009:66:854-9.

21 Department of Health Social Services and Public Safety (DHSSPS) McKinsley Report. Reshaping the system: Implications for Northern Ireland Health and Social Care Service of the 2010 spending review. DHSSPS, 2010

22 Luo R, Scullin C, McElnay JC, et al. One stop dispensing and discharge prescription time. Hospital Pharmacy Europe 2012;65:43-6.

23 Hogg A, Scullin C, Luo R, et al. Do patient bedside medicine lockers result in a safer and faster medicine administration round? Eur J Hosp Pharm 2012;19:525-8.

24 McCrudden M, Magee FA, Farren D, et al. IV antibiotic prescribing in surgical patients - an audit. Hosp Pharm Eur 2013:71:36-7.

25 Maripuu $H$, Aldeyab MA, Kearney MP, et al. Respiratory and urinary tract infections in a hospital setting. Eur J Hosp Pharm 2014:21:139-44.

26 El Hajji FWD, Scullin C, Scott MG, et al. Enhanced Clinical Pharmacy Service targeting tools risk predictive algorithm. J Eval Clin Pract doi: 10.1111/jep.12276 Sep 2014

27 Lynas R, Hunter A, Gill D, et al. Keyless locker helps staff and patients. Pharm J 2005;273:294-5.

28 Scott MG, McElnay JC, Janknegt R, et al. Safe Therapeutic Economic Pharmaceutical Selection (STEPSelect): development, introduction and use in Northern Ireland. EJHP-P 2010;16:81-3.

29 Janknegt R, Scott M, Mairs J, et al. System of Objectified Judgement Analysis (SOJA) as a tool in rational and transparent drug decision-making. Expert Opin Pharmacother 2007:8(Suppl 1):S5-S14.

30 Beagon P, Martin M, Scott MG. Secure wireless access to a patient's administration system (PAS) and real-time intervention recording using handheld computers. 5th European Society for Clinical Pharmacy Meeting; Stockholm, May 2005.

31 Scott MG, McElnay JC and Burnett KM. Using barcode technology to capture electronic intervention data in a hospital with a stand-alone pharmacy computer system. Am J Health Syst Pharm 1996:53:651-4.

32 Scullin C, Hogg A, Luo R, et al. Integrated medicines management-can routine implementation improve quality? J Eval Clin Pract 2012;18:807-15.

33 Gillespie U, Alassaad A, Henrohn D, et al. A comprehensive pharmacist intervention to reduce morbidity in patients 80 years or older. Arch Intern Med 2011;169:894-900.

34 Hellstrom LM, Bondeson A, Hoglund P, et al. Impact of the Lund Integrated Medicines Management (LIMM) model on medication appropriateness and drug related hospital readmissions. Eur J Clin Pharmacol 2011;67:741-52.

35 Grimes T, Deasy E, Allen A, et al. Collaborative pharmaceutical care in an Irish Hospital: uncontrolled before-after study. BMJ Qual Saf 2014;23:574-83.

36 Anderson $\mathrm{AH}$, Wedre LJ, Kutschera Sund J, et al. Evaluation of implementation of clinical pharmacy service in Central Norway. Eur J Hosp Pharm 2014;21: 125-8

37 Cochrane $R$, Mandal $A$, Ledger-Scott $M$, et al. Changes in drug treatment after discharge from hospital in geriatric patients. BMJ 1992:305:694-6.

38 Barker A, Travis E. Re-engineering medicines supply arrangements at Ipswich Hospital—were we mad? Pharmacy Management 2005;21:9-14.

39 Campbell D, Conroy C, Cameron T, et al. One Stop or Non-stop? Assessing the benefits of near patient dispensing on a general medical ward. Int J Pharm Pract 2003; 11:R87.

40 Armitage $\mathrm{G}$, Krampira $\mathrm{H}$. Adverse events in drug administration; a literature review. J Nurs Manag 2003:11:130-40.

41 Tissot $E$, Cornette $C$, Demoly $P$, et al. Medication errors at the administration stage in an intensive care unit. Intensive Care Med 1999:25:353-9.

42 Van den Bemt PM, Fijn R, Van der Voort PH, et al. Frequency and determination of drug administration errors in the intensive care unit. Crit Care Med 2002;30:846-50

43 Lisby M, Nielsen LP, Mainz J. Errors in the medication process: frequency, type and potential clinical consequences. Int J Qual Health Care 2005;17:15-22.

44 Franklin BD, O'Grady K, Voncina L, et al. An evaluation of two automated dispensing machines in UK hospital pharmacy. Int J Pharm Pract 2008;16:47-53.

45 Bennett J, Harper-Femson LA, Tone J, et al. Improving medication administration systems: an evaluation study. Can Nurse 2006;102:35-9.

46 Ramirez JA. Early switch from intravenous to oral antibiotics and early hospital discharge: a prospective observational study of 200 consecutive patients with community acquired pneumonia. Arch Intern Med 1999;159:2449-54. 


\section{Original article}

47 Aldeyab MA, Kearney MP, McElnay JC, et al. A point prevalence survey of antibiotic prescription benchmarking and patterns of use. Br J Clin Pharmacol 2011;71:293-6.

48 Billings J, Doxon J, Mijanovich T, et al. Core findings for patients at risk of readmission to hospital: development of an algorithm to identify high risk patients. BMJ 2006;333:32-3.

49 Bond CA, Raehl CL, Franke T. Interrelationships among mortality rates, drugs costs, total cost of care and length of stay in: United States Hospital. Summany and recommendation for clinical pharmacy services and staffing. Pharmacotherapy 2001;21:129-41.

50 Bond CA, Raehl CL. 2006 National clinical pharmacy services survey. Clinical pharmacy services collaborative: drug management, medication errors and pharmacy technology. Pharmacotherapy 2008;28:1-13.
51 Bond CA, Raehl CL. Clinical pharmacy services, pharmacy staffing and hospital mortality rates. Pharmacotherapy 2007;27:481-93.

52 Miller R, Darcy C, Friel A, et al. Pharmacist Care Management Service of Elderly Patients in Intermediate Care. FIP 2013. Towards a Future Vision for Complex Patients http://www. fip.org/abstracts?page=abstracts\&actions=item\& item8829

53 Karnon J, Campbell F, Czoski-Murray C. Modelling the expected net benefits of interventions to reduce the burden of medication errors. I Health Serv Res Policy 2008;13:85-91.

54 European Innovation Partnership on Active and Health Ageing Reference SitesExcellence Innovation for Ageing A European Guide Brussels. 2013. 